\title{
SHORT REPORT \\ Detection of HEV-specific antibodies in four non-human primate species, including great apes, from different zoos in Germany
}

\author{
C. SPAHR ${ }^{1,2}$, T. KNAUF-WITZENS ${ }^{1}$, L. DÄHNERT ${ }^{3}$, M. ENDERS ${ }^{4}$, \\ M. MÜLLER ${ }^{4}$, R. JOHNE ${ }^{5}$ AND R. G. ULRICH ${ }^{3,6 *}$ \\ ${ }^{1}$ Wilhelma Zoological-Botanical Gardens, Stuttgart, Germany \\ ${ }^{2}$ Faculty of Veterinary Medicine, Institute of Virology, University of Leipzig, Leipzig, Germany \\ ${ }^{3}$ Friedrich-Loeffler-Institut, Federal Research Institute for Animal Health, Institute of Novel and Emerging \\ Infectious Diseases, Greifswald-Insel Riems, Germany \\ ${ }^{4}$ Laboratory Enders, Prof. Dr. med. Gisela Enders \& Partners, Stuttgart, Germany \\ ${ }^{5}$ German Federal Institute of Risk Assessment, Berlin, Germany \\ ${ }^{6}$ German Center for Infection Research (DZIF), Partner site Hamburg-Luebeck-Borstel-Insel Riems, Germany
}

Received 18 May 2017; Final revision 20 October 2017; Accepted 25 October 2017;

first published online 23 November 2017

\section{SUMMARY}

The hepatitis E virus (HEV) has been described in humans and various animal species in different regions of the world. However, the knowledge on natural HEV infection in non-human primates and the corresponding risk of zoonotic transmission is scarce. To determine whether primates in captivity are affected by HEV infection, we investigated 259 individual sera of clinically healthy non-human primates of 14 species from nine German zoos. Using a commercial double-antigen-sandwich ELISA and a commercial IgG ELISA, 10 animals (3.9\%) reacted positive in at least one assay. Three ape species and one Old World monkey species were among the seropositive animals: bonobo (Pan paniscus), gorilla (Gorilla gorilla gorilla), lar gibbon (Hylobates lar) and drill (Mandrillus leucophaeus). Testing for anti-HEV-IgM antibodies by commercial ELISA and for viral RNA by reverse-transcription real-time polymerase chain reaction resulted in negative results for all animals indicating the absence of acute HEV infections. In the past, no clinical signs of hepatitis were recorded for the seropositive animals. The results suggest that non-human primates in zoos can get naturally and subclinically infected with HEV or related hepeviruses. Future studies should evaluate potential sources and transmission routes of these infections and their impact on human health.

Key words: ELISA, hepatitis E virus, human, non-human primates, seroconversion, zoonosis.

Hepatitis $\mathrm{E}$ is a worldwide occurring, notifiable emerging infectious disease caused by hepatitis $\mathrm{E}$ virus (HEV), which comprises different genotypes with

\footnotetext{
* Author for correspondence: R. G. Ulrich, Friedrich-LoefflerInstitut, Federal Research Institute for Animal Health, Institute of Novel and Emerging Infectious Diseases, 17493 Greifswald-Insel Riems, Germany.

(Email: rainer.ulrich@fli.de)
}

different transmission modes and geographic distributions [1]. The number of recorded human cases has steadily been increasing in several European countries during the past 10 years [2]. HEV-RNA and anti-HEVantibodies have also been detected in a considerable variety of wild-living, farmed and pet animal species worldwide. Additional hepeviruses from animals have been discovered in previous years, mostly with unknown zoonotic potential [1]. 
Recently, a novel taxonomical classification of hepeviruses was introduced [3]. HEV belongs to the family Hepeviridae, which taxonomically is divided into two genera: Orthohepevirus and Piscihepevirus. The genus Orthohepevirus contains all mammalian and avian HEV genotypes. The genus Piscihepevirus only contains the HEV-like agent from cutthroat trout (Oncorhynchus clarki) and related fish species. The species Orthohepevirus A comprises genotypes HEV-1 to HEV-4 and HEV-7 which are human pathogenic. Out of these, HEV-3, HEV-4 and HEV-7 are zoonotic pathogens, with wild boar, domestic pig, rabbit, deer and dromedary representing their reservoirs. In contrast, little is known about HEV infections in non-human primates. Experimental infections with human-pathogenic, zoonotic HEV-3 were successful in Japanese macaques (Macaca fuscata) and rhesus macaques (Macaca mulatta) [4]. Primates kept in zoos are in close contact with their keepers and are known to be susceptible for diverse human pathogens, like influenza, herpes or hepatitis B viruses [5-7]. Therefore, the objective of our study was to investigate if $\mathrm{HEV}$ infections occur within primate populations in zoos.

Within the years 2015 and 2016, a total of 259 individual sera of 14 non-human primate species were collected from nine German zoos. The sera were obtained during immobilisations for different purposes or taken from serum collections of the participating zoos. Full blood was centrifuged and the supernatant stored at $-20{ }^{\circ} \mathrm{C}$ until further use. The physical health status of all animals was routinely checked and documented by their keepers on a daily basis.

Three commercial anti-HEV antibody ELISAs were performed and rated according to the protocols of the manufacturers: Axiom $^{\circledR}$ HEV-Ab-EIA (Axiom Diagnostik, Bürstadt, Germany), Mikrogen ${ }^{\circledR}$ recomWell HEV-IgG and Mikrogen ${ }^{\circledR}$ recomWell HEV-IgM (both Mikrogen Diagnostik, Neuried, Germany). The double antigen sandwich-based Axiom assay detects all classes of antibodies and is based on a recombinant open reading frame 2 (ORF2)-derived antigen of HEV-1. The other two assays are specific for IgG and IgM and are based on recombinant ORF2-derived antigens of HEV-1 and HEV-3. The 95\% confidence intervals (CIs) were calculated in $\mathrm{R}$ (v3.4.0) using the binom.confint function of the binom package (exact method). For Axiom $^{\circledR}$ HEV-Ab-ELISA, serum samples were diluted $1: 1$, for initially positive samples serial dilutions were tested $(1: 10,1: 50,1: 250,1: 1250)$. For
Mikrogen ${ }^{\circledR}$ recomWell HEV-IgG and -IgM assays, serum samples were diluted $1: 101$. RNA was extracted from serum using a commercial kit following the manufacturer's instructions (NucleoMag ${ }^{\mathbb{B}}$ VET MACHEREY-NAGEL, Düren, Germany). One hundred microlitres of each clarified sample was used for RNA extraction. During RNA isolation, RNA bacteriophage MS2 was added as an indicator for successful extraction [8]. Finally, the RNA was stored at $-80^{\circ} \mathrm{C}$ until further use.

A reverse-transcription real-time polymerase chain reaction (RT-qPCR) was performed with 256 samples, using the QuantiTect Probe ${ }^{\circledR}$ RT-PCR kit (QIAGEN $\mathrm{GmbH}$, Hilden, Germany) in a $20 \mu$ reaction volume with primer concentration of $1 \mu \mathrm{M}$. For negative and positive controls, $5 \mu \mathrm{l}$ of RNase-free water and $5 \mu \mathrm{l}$ of HEV-RNA from a domestic pig, corresponding to $3 \cdot 7$ genome copies, were used. The RT-qPCR followed the thermal profile of a standard protocol with 45 cycles [9]. The evaluation of the RT-qPCR results followed the published standard protocol. The RT-qPCR was performed in a multiplex format to detect simultaneously HEV and phage MS2 RNA. All samples were positive for phage MS2 RNA confirming successful RNA extraction.

A total of 259 individual serum samples were tested for the presence of anti-HEV antibodies, using the Axiom ${ }^{\circledR}$ HEV-Ab-EIA (Table 1). Seven of $259(2 \cdot 7 \%$, $95 \%$ CI $1 \cdot 1-5 \cdot 5 \%$ ) sera were anti-HEV positive, including four gorillas (Gorilla gorilla gorilla), one bonobo (Pan paniscus), one lar gibbon (Hylobates lar) and one drill (Mandrillus leucophaeus). The positive samples originated from five of the nine zoos (A, B, F, G and H) (Supplementary Tables S1 and S2). In total, 257 of the 259 samples were additionally tested using the Mikrogen ${ }^{\circledR}$ recomWell ELISAs, as for two of the 259 samples, there was no more material available. Six of 257 sera $(2 \cdot 3 \%, 95 \%$ CI $0 \cdot 9-5 \%)$ were positive $(n=4)$ or equivocal $(n=2)$ for anti-HEV-IgG; all six samples originated from gorillas. For both tests combined, the overall seroprevalence in gorillas was $15 \cdot 2 \%(95 \%$ CI 6.3-28.9\%; Table 1), whereas the seroprevalence for this species based on the single tests was $8.7 \%(95 \%$ CI $2 \cdot 4-20 \cdot 8 \%)$ and $13 \%(95 \%$ CI $4 \cdot 9-26 \cdot 3 \%)$ for Axiom ${ }^{\circledR}$ HEV-Ab-EIA and Mikrogen ${ }^{\circledR}$ recomWell HEV-IgG-ELISA, respectively (Table 1). Three of the gorilla samples were seroreactive in both tests (Supplementary Table S1). One of these three samples was also reactive in the Axiom ${ }^{\circledR} \mathrm{HEV}$-Ab-EIA at higher dilutions (Supplementary Table S3). In contrast, one bonobo, one lar gibbon and one drill were exclusively 
Table 1. Prevalence of anti-hepatitis E virus antibodies in non-human primates from nine zoos in Germany determined by two ELISAs

\begin{tabular}{|c|c|c|c|c|c|c|c|c|c|c|c|}
\hline \multirow[b]{2}{*}{ Family } & \multirow[b]{2}{*}{ Species } & & \multicolumn{3}{|c|}{ Axiom ${ }^{\circledR}$ HEV-Ab-EIA } & \multicolumn{3}{|c|}{$\begin{array}{l}\text { Mikrogen }{ }^{\circledR} \text { recomWell } \\
\text { HEV-IgG }\end{array}$} & \multicolumn{3}{|c|}{$\begin{array}{l}\text { No. individuals pos. or equi } \\
\text { in at least one assay/total no. } \\
\text { individuals investigated }\end{array}$} \\
\hline & & & pos./total & $\%$ & $95 \% \mathrm{CI}$ & pos./total & $\%$ & $95 \% \mathrm{CI}$ & pos./total & $\%$ & $95 \% \mathrm{CI}$ \\
\hline \multirow[t]{5}{*}{ Hominidae } & Gorilla & Gorilla gorilla gorilla & $4 / 46$ & $8 \cdot 7$ & $2 \cdot 4-20 \cdot 8$ & $6 / 46$ & 13 & $4 \cdot 9-26 \cdot 3$ & $7 / 46$ & $15 \cdot 2$ & $6 \cdot 3-28 \cdot 9$ \\
\hline & Bonobo & Pan paniscus & $1 / 25$ & $4 \cdot 0$ & $0 \cdot 1-20 \cdot 4$ & $0 / 25$ & - & $0-13 \cdot 7$ & $1 / 25$ & $4 \cdot 0$ & $0 \cdot 1-20 \cdot 4$ \\
\hline & Chimpanzee & Pan troglodytes & $0 / 70$ & - & $0-5 \cdot 1$ & $0 / 70$ & - & $0-5 \cdot 1$ & $0 / 70$ & - & $0-5 \cdot 1$ \\
\hline & Sumatran orangutan & Pongo abelii & $0 / 16$ & - & $0-20 \cdot 6$ & $0 / 15$ & - & $0-21 \cdot 8$ & $0 / 16$ & - & $0-20 \cdot 6$ \\
\hline & Bornean orangutan & Pongo pygmaeus & $0 / 4$ & - & $0-60 \cdot 2$ & $0 / 4$ & - & $0-60 \cdot 2$ & $0 / 4$ & - & $0-60 \cdot 2$ \\
\hline Hylobatidae & Lar gibbon & Hylobates lar & $1 / 11$ & $9 \cdot 1$ & $0 \cdot 2-41 \cdot 3$ & $0 / 11$ & - & $0-28,5$ & $1 / 11$ & $9 \cdot 1$ & $0 \cdot 2-41 \cdot 3$ \\
\hline \multirow[t]{5}{*}{ Cercopithecidae } & Drill & Mandrillus leucophaeus & $1 / 7$ & $14 \cdot 3$ & $0 \cdot 4-57 \cdot 9$ & $0 / 7$ & - & $0-41$ & $1 / 7$ & $14 \cdot 3$ & $0 \cdot 4-57 \cdot 9$ \\
\hline & Gelada baboon & Theropithecus gelada & $0 / 61$ & - & $0-5 \cdot 9$ & $0 / 61$ & - & $0-5 \cdot 9$ & $0 / 61$ & - & $0-5 \cdot 9$ \\
\hline & Javan silvered leaf monkey & Trachypithecus auratus & $0 / 6$ & - & $0-45 \cdot 9$ & $0 / 6$ & - & $0-45 \cdot 9$ & $0 / 6$ & - & $0-45 \cdot 9$ \\
\hline & Japanese macaque & Macaca fuscata & $0 / 2$ & - & $0-84 \cdot 2$ & $0 / 2$ & - & $0-84 \cdot 2$ & $0 / 1$ & - & $0-97 \cdot 5$ \\
\hline & White-crowned mangabey & Cercocebus atys lunulatus & $0 / 1$ & - & $0-97 \cdot 5$ & $0 / 0$ & - & - & $0 / 1$ & - & $0-97 \cdot 5$ \\
\hline \multirow[t]{2}{*}{ Atelidae } & Black howler monkey & Alouatta caraya & $0 / 3$ & - & $0-70 \cdot 8$ & $0 / 3$ & - & $0-70 \cdot 8$ & $0 / 3$ & - & $0-70 \cdot 8$ \\
\hline & White-fronted spider monkey & Ateles hybridus & $0 / 4$ & - & $0-60 \cdot 2$ & $0 / 4$ & - & $0-60 \cdot 2$ & $0 / 4$ & - & $0-60 \cdot 2$ \\
\hline Cebidae & Black-capped squirrel monkey & Saimiri boliviensis & $0 / 1$ & - & $0-97 \cdot 5$ & $0 / 1$ & - & $0-97 \cdot 5$ & $0 / 1$ & - & $0-97 \cdot 5$ \\
\hline Pitheciidae & White-faced saki & Pithecia pithecia & $0 / 2$ & - & $0-84 \cdot 2$ & $0 / 2$ & - & $0-84 \cdot 2$ & $0 / 2$ & - & $0-84 \cdot 2$ \\
\hline Total & & & $7 * / 259$ & $2 \cdot 7$ & $1 \cdot 1-5 \cdot 5$ & $6 * * / 257$ & $2 \cdot 3$ & $0 \cdot 9-5$ & $10 / 259$ & 3.9 & $1 \cdot 9-7$ \\
\hline
\end{tabular}

pos., positive; total, total number of samples analysed; equi., equivocal; $95 \% \mathrm{CI}, 95 \%$ confidence interval; $7 *$, result contains $6 / 172(3 \cdot 5 \%)$ apes and $1 / 87(1 \cdot 2 \%)$ of the residue monkeys; $6^{* *}$, result contains four pos. and two equivocal samples from gorillas. 
reactive in the Axiom ${ }^{\circledR}$ HEV-Ab-EIA, but not in the Mikrogen ${ }^{\circledR}$ recomWell HEV-IgG-ELISA. None of the orangutans (Pongo abelii and Pongo pygmaeus) and chimpanzees (Pan troglodytes) showed anti-HEV-antibodies in any of the applied tests (Table 1). In addition, none of the investigated samples were positive for anti-HEV-IgM or HEV-RNA-positive in the RT-qPCR (data not shown).

Here we describe a HEV seroprevalence study in non-human primates from nine zoos in Germany. HEV-reactive antibodies were detected in 10 of 259 (3.9\%; 95\% CI 1.9-7\%) individuals, which represents a low seroprevalence compared with the results of serosurveys in humans, wild boar or pigs in Germany $[10,11]$. None of the seropositive animals displayed signs of an acute infection at the time of sampling, as evidenced by the absence of HEV-RNA and HEV-specific IgM antibodies. The absence of these parameters of an acute infection might be caused by a lower sensitivity of the IgM assay, licensed for human diagnostics, or by the high specificity of the RT-qPCR assay, that probably is unable to detect RNA of HEV cross-reactive, but different, so far unknown hepeviruses. In line with the absence of descriptions of natural HEV infection in non-human primates displaying clinical illness, the seroreactive animals in this study had never been reported to show hepatitis E-related clinical symptoms in the past. Three species of apes, including two great ape species and one lar gibbon, plus one Old World monkey species were found to be anti-HEV antibody positive. Interestingly, none of the 70 chimpanzees and 61 gelada baboons (Theropithecus gelada) were found to be anti-HEV positive, including 17 chimpanzees and all gelada baboons from zoo ' $\mathrm{H}$ ', where HEV-specific antibodies were detected in four gorillas, one bonobo and one drill (Supplementary Table S2). The observed local variation in HEV seroprevalence may be most likely caused by a sampling bias, as most samples $(166 / 259,64 \cdot 1 \%)$ were collected from zoo 'H' (see Supplementary Table S2).

The number of non-human primates tested for natural HEV infection is scarce so far, but the reported prevalences were mostly higher. An investigation of 92 sera from wild rhesus macaques in the rural city of Kunming, Yunnan Province, China, resulted in a HEV-IgG-seroprevalence of $35 \cdot 87 \%$ [12]. As many pigs and wild boars are housed in Yunnan Province, the authors of this study suggested that contact of monkeys and swine may have led to cross-species transmission of HEV-4. Furthermore, a HEV-3 outbreak in a Japanese outdoor breeding monkey facility was reported [4]. The afflicted animals were Japanese macaques and rhesus macaques. Within a period of 5 years, the HEV-IgG-seroprevalence ranged between 20\% (2005), 78.5\% (2006) and 35.3\% (2009). During this time, no clinical hepatitis E-like symptoms were observed, neither in the macaques, nor in the staff. The source of HEV infection in this study could not be identified. Natural HEV infection in great apes was reported only once; in a Chinese zoo, HEV-RNA was detected in $29 \cdot 2 \%$ (7/24) of faecal samples from chimpanzees [13]. Phylogenetic analyses suggested the virus strain to represent a novel HEV type, but its taxonomical classification is still pending.

The source and route of transmission of HEV or a related hepevirus in the animals investigated here and the potential reason for the observed difference in seroprevalence between gorillas $(7 / 46 ; 15 \cdot 2 \%, 95 \%$ CI $6 \cdot 3-$ $28 \cdot 9)$ and chimpanzees $(0 / 70 ; 0 \%, 95 \%$ CI $0-5 \cdot 1)$ remain obscure, but might be also due to sampling bias. Three old gorillas were wild born (individuals no. 1, 4, 7) and it cannot be excluded that they got infected during their earlier life in the wild. In these cases, direct contact to infected group members from the same species, or contact with faecal excretions or contaminated water or food may portray a risk for infection. In contrast, seven seroreactive animals were born in captivity, indicating that they must have been infected in a zoo. Direct contact to the keepers cannot be ruled out as a source and route of transmission. Even though hygiene standards in the holding institutions were high and pest control programmes enforced, contact to wild rabbits or wild rats in outdoor enclosures, either direct or via excretions, poses a potential route of transmission. As gorillas kept in zoos are strictly fed vegetarian, foodborne infection via contaminated meat seems to be unlikely, although wild gorillas, similar to chimpanzees, have been observed to consume wild caught mice and rats. In addition, housing swine and primates in the same zoo may be suspected to enable indirect transmission of HEV via faecally contaminated shoes, utensils or vehicles, carried by the personnel. Interestingly, we investigated two anti-HEV-seroreactive female gorillas from zoo ' $\mathrm{H}$ ' (Supplementary Table S1), being mother (no. 4) and her 19-year-old child (no. 6). Vertical transmission and breast-feeding may be further possible explanations for HEV infections in great apes, too. The presence of maternal antibodies in the younger animal can be excluded as the animal was already 19 years old during the time of sample collection. 
As reflected in Supplementary Table S1, results of the Axiom ${ }^{\circledR}$ HEV-Ab-EIA and Mikrogen ${ }^{\circledR}$ recomWell HEV-IgG-ELISA are only partially matching with the latter assay showing a lower seroprevalence. This observation is in line with previous investigations in humans $[14,15]$ and pigs $[16,17]$ and might be caused by the different test principles used [10,11, 18], but also partially influenced by the use of an IgG assay adapted for human sera. In addition, the long-term storage and frequent freeze-thaw cycles of the samples from serum banks might have resulted in a decrease of antibody amounts in the serum samples.

In conclusion, the present study indicates that nonhuman primates in zoos are susceptible to natural infection with HEV or related hepeviruses. The observed seroprevalences were found to be very low. This low prevalence in primates is in contrast to the assumption of an anthropozoonotic transmission from non-human primates to humans, but may underline the transmission of HEV by consumption of contaminated groceries or direct contact of humans to domestic pigs. In particular, gorillas were afflicted more often than other apes or non-human primates. Further investigations are needed to prove potential differences in the susceptibility of certain primate species, to identify the hepevirus origin of their infections and the potential transmission routes and to evaluate the veterinary and public health risk consequences. For this purpose, future HEV monitoring in nonhuman primates and potential reservoir species, such as pigs, deer, rabbits and rats, in zoos is highly recommended.

\section{SUPPLEMENTARY MATERIAL}

The supplementary material for this article can be found at https://doi.org/10.1017/S0950268817002606.

\section{ACKNOWLEDGEMENTS}

We thank all colleagues from the cooperating zoos for the sample material, Thomas W. Vahlenkamp and Martin H. Groschup for continuous support and Birgit Schauer and Andreas Fröhlich for excellent statistical advice and calculation of the $95 \%$ CI values.

This study was supported with a scholarship by the 'Grimminger Stiftung für Zoonosenforschung' (GSZ), Germany. The research within the laboratory of Dr Rainer G. Ulrich was supported by the German Centre for Infection Research (DZIF).

\section{DECLARATION OF INTEREST}

None.

\section{REFERENCES}

1. Johne R, et al. Hepeviridae: an expanding family of vertebrate viruses. Infection, Genetics and Evolution 2014; 27(2014): 212-229. Available from: doi: 10.1016/j. meegid.2014.06.024.

2. Adlhoch C, et al. Hepatitis E virus: assessment of the epidemiological situation in humans in Europe, 2014/ 15. Journal of Clinical Virology 2016; 82: 9-16.

3. Smith DB, et al. Consensus proposals for classification of the family Hepeviridae. Journal of General Virology 2014; 95: 2223-2232.

4. Yamamoto H, et al. Hepatitis E virus outbreak in monkey facility, Japan. Emerging Infectious Diseases 2012; 18(12): 2032-2034.

5. Buitendijk H, et al. Retrospective serology study of respiratory virus infections in captive great apes. Viruses 2014; 6(3): 1442-1453.

6. Meals LC, et al. Prospective population management for hepatitis B in the chimpanzee (Pan troglodytes) $\mathrm{Ssp}^{\circledR}$ population. Journal of Zoo and Wildlife Medicine 2016; 47(3): 711-716. Available from: http:// www.bioone.org/doi/10.1638/2015-0237.1.

7. Fickenscher H, Fleckenstein B. Herpesvirus saimiri. Royal Society 2001; 356(1408): 545-567. Available from: http://www.pubmedcentral.nih.gov/articlerender. fcgi?artid=1088444\&tool=pmcentrez\&rendertype $=$ abstract.

8. Hammerschmidt F, et al. Hepatitis E virus in wild rabbits and European brown hares in Germany. Zoonoses and Public Health 2017; 11(2016): 1-11. Available from: http://doi.wiley.com/10.1111/zph.12355.

9. Schlosser J, et al. Natural and experimental hepatitis E virus genotype 3-infection in European wild boar is transmissible to domestic pigs. Veterinary Research 2014; 45(121): 1-13.

10. Dremsek $\mathbf{P}$, et al. Seroprevalence study in forestry workers from eastern Germany using novel genotype 3- and rat hepatitis $E$ virus-specific immunoglobulin $G$ ELISAs. Medical Microbiology and Immunology 2012; 201(1): 189-200.

11. Dremsek P, et al. Hepatitis E virus seroprevalence of domestic pigs in Germany determined by a novel in-house and two reference ELISAs. Journal of Virological Methods 2013; 190: 11-16.

12. Huang F, et al. Seroepidemiology and molecular characterization of hepatitis E virus in Macaca mulatta from a village in Yunnan, China, where infection with this virus is endemic. Hepatitis Monthly 2011; 11(9): $745-749$.

13. Zhou C, Li W, Yang S. Analysis of hepatitis E virus-like sequence in Chimpanzee. Hepatitis Monthly 2014; 14 (9): 2.

14. Krumbholz A, et al. Seroprevalence of hepatitis E virus (HEV) in humans living in high pig density areas of 
Germany. Medical Microbiology and Immunology 2014; 203(4): 273-282.

15. Wenzel JJ, et al. Test performance characteristics of anti-HEV IgG assays strongly influence hepatitis E seroprevalence estimates. Journal of Infectious Diseases 2013; 207(3): 497-500.

16. Baechlein C, et al. Prevalence of hepatitis E virus-specific antibodies in sera of German domestic pigs estimated by using different assays. Veterinary Microbiology 2010; 144 (1-2): 187-191. Available from: doi: 10.1016/j.vetmic. 2009.12.011.
17. Krumbholz A, et al. Age-related and regional differences in the prevalence of hepatitis E virus-specific antibodies in pigs in Germany. Veterinary Microbiology 2013; 167 (2013): 394-402.

18. Sommerkorn FM, et al. Performance of hepatitis E virus (HEV)-antibody tests: a comparative analysis based on samples from individuals with direct contact to domestic pigs or wild boar in Germany. Medical Microbiology and Immunology 2017; 206(3): 277-286. Available from: http://link.springer.com/10.1007/s00430017-0503-4. 